\title{
MEMBERS OF THE SOCIETY
}

\author{
OCTOBER, 1928
}

\section{Sustaining Members}

Aetna Life Insurance Company, Hartford, Conn.

Allyn AND Bacon, Boston, Mass.

Amherst College, Amherst, Mass.

BABCOCK AND WILCOX CoMPANY, New York, N. Y.

Bell Telephone Laboratories, New York, N. Y. Patron.

Connecticut General Life Insurance Company, Hartford, Conn.

Dartmouth College, Hanover, N. H.

Eastman Kodak Company, Rochester, N. Y.

Edison Electric Illuminating Company, Boston, Mass.

Equitable Life Insurance Company of Iowa, Des Moines, Ia.

General Electric Company, Schenectady, N. Y. Patron.

Georgia Power Company, Atlanta, Ga.

Ginn AND Company, Boston, Mass.

HaRvard University, Cambridge, Mass.

InTERnational Life Insurance Company of St. Louis, St. Louis, Mo.

John Hancock Mutual Life Insurance Company, Boston, Mass.

Massachusetts Institute of TeChnology, Cambridge, Mass.

Mathematical Association of America, Oberlin, Ohio.

Metropolitan Life Insurance Company, New York, N. Y.

Missouri State Life Insurance Company, St. Louis, Mo.

National Life Insurance Company, Montpelier, Vt.

National Life Insurance Company of the United States of America, Chicago, Ill.

New England Mutual Life Insurance Company, Boston, Mass.

New York Edison CoMpany, New York, N. Y.

NORTHWESTERN UNIVERSITY, Evanston, III.

Pacific Mutual Life Insurance Company, Los Angeles, Calif.

Penn Mutual Life Insurance Company, Philadelphia, Pa.

Phoenix Mutual Life Insurance Company, Hartford, Conn.

Prudential Insurance Company, Newark, N. J.

Mr. E. W. RICE, JR., General Electric Company, Schenectady, N. Y.

SARGENT AND LUNDY, Chicago, Ill.

Travelers Insurance Company, Hartford, Conn.

Union Central Life Insurance Company, Cincinnati, Ohio.

University of Michigan, Ann Arbor, Mich.

UnIversity of Pennsylvania, Philadelphia, $\mathrm{Pa}$.

UNIVERSITY OF WASHINGTON, Seattle, Wash.

UNIVERSITY OF WisconsIN, Madison, Wis.

Western and Southern Life Insurance Company, Cincinnati, Ohio. 